\title{
A SERENDIPITOUS ALL SKY SURVEY FOR BRIGHT OBJECTS IN THE OUTER SOLAR SYSTEM
}

\author{
M. E. Brown ${ }^{1}$, M. T. Bannister ${ }^{2,3}$, B. P. Schmidt $^{3}$, A. J. Drake ${ }^{1}$, S. G. Djorgovski ${ }^{1}$, M. J. Graham ${ }^{1}$, A. Mahabal ${ }^{1}$, \\ C. Donalek ${ }^{1}$, S. Larson $^{4}$, E. Christensen ${ }^{4}$, E. Beshore ${ }^{4}$, and R. McNaught ${ }^{3}$ \\ ${ }^{1}$ California Institute of Technology, Pasadena, CA, USA \\ ${ }^{2}$ University of Victoria, Victoria, BC, Canada \\ ${ }^{3}$ The Australian National University, Canberra, Australia \\ ${ }^{4}$ The University of Arizona, Lunar and Planetary Laboratory, Tucson, AZ, USA \\ Received 2014 September 16; accepted 2014 December 8; published 2015 January 19
}

\begin{abstract}
We use seven year's worth of observations from the Catalina Sky Survey and the Siding Spring Survey covering most of the northern and southern hemisphere at galactic latitudes higher than $20^{\circ}$ to search for serendipitously imaged moving objects in the outer solar system. These slowly moving objects would appear as stationary transients in these fast cadence asteroids surveys, so we develop methods to discover objects in the outer solar system using individual observations spaced by months, rather than spaced by hours, as is typically done. While we independently discover eight known bright objects in the outer solar system, the faintest having $V=19.8 \pm 0.1$, no new objects are discovered. We find that the survey is nearly $100 \%$ efficient at detecting objects beyond $25 \mathrm{AU}$ for $V \lesssim 19.1$ ( $V \lesssim 18.6$ in the southern hemisphere) and that the probability that there is one or more remaining outer solar system object of this brightness left to be discovered in the unsurveyed regions of the galactic plane is approximately $32 \%$.
\end{abstract}

Key words: Kuiper belt: general - planets and satellites: detection - planets and satellites: formation

\section{INTRODUCTION}

The last decade has seen the discovery of most of the brightest objects in the outer solar system (Trujillo \& Brown 2003; Brown 2008; Schwamb et al. 2009). The widefield surveys for these brightest objects appear moderately complete in both the northern and southern skies, with only the generally avoided galactic plane and ecliptic poles left to survey completely. All surveys miss some fraction of objects ostensibly in their survey regions, however, due to temporal gaps, detector gaps, stellar blending, and numerous other difficulties. Survey efficiencies have been estimated to be between 70\% and 90\% (Schwamb et al. 2010; Sheppard et al. 2011; Rabinowitz et al. 2012), leaving the possibility that bright objects in the outer solar system have escaped detection.

While each of the bright objects in the Kuiper belt has yielded an important boon of scientific information about the origin and evolution of the Kuiper belt and its objects (Brown 2008), mounting a dedicated survey to find this small number of remaining bright objects would be prohibitive. We note, however, that each of the bright objects discovered over the past decade serendipitously appeared in multiple other survey images. These serendipitous detections have been reported to the Minor Planet Center in sources from the Skymorph data of CCD images from the Palomar 48 inch Schmidt ${ }^{5}$ back to the original POSS I photographic plates of the 1950s and many in between. The objects were unrecognized at the time of their original imaging owing to the fact that even at opposition these objects move at speeds of only a few arcseconds per hour, so they appear identical to stationary stars in the images. Only by comparing the images with archival images of the same location taken at a different time is it recognized that the bright outer solar system object appears as a one-time transient.

http://skyview.gsfc.nasa.gov/skymorph/
The large number of serendipitous images of bright outer solar system objects raises the possibility that a fully serendipitous archival survey could be attempted to find new objects. An ideal data set for such a survey would be one which covers large areas of the sky with high enough temporal coverage that multiple detection of an object in the Kuiper belt could be made.

Over the past decade, surveys for NEOs have come closest to achieving this ideal. In these surveys multiple images are obtained to search for moving objects, but these images are obtained over a time period too short to detect the motion of slowly moving objects in the outer solar system. Objects in the outer solar system appear simply as stationary transients.

Here we describe a serendipitous all sky survey for bright objects in the outer solar system using archival data from the Catalina Sky Survey (CSS) in the northern hemisphere and its sister survey, the Siding Spring Survey (SSS), in the southern hemisphere.

\section{THE CSS, SSS, AND CRTS SURVEYS}

The CSS (Larson et al. 2003) operates on the $0.7 \mathrm{~m}$ Catalina Schmidt telescope at the Catalina Observatory in Arizona and covers 8.1 square degrees per field to a limiting magnitude of $V \sim 19.5$. The CSS has covered approximately 19,700 square degrees of sky between decl. of -25 and +70 at galactic latitudes greater than $10^{\circ}$. The SSS operates on the $0.5 \mathrm{~m}$ Uppsala Schmidt telescope at Siding Spring Observatory in Australia and covers 4.2 square degrees per field to a limiting magnitude of approximately 19.0. The SSS has covered approximately 14,100 square degrees of sky from decl. -80 to 0. Accounting for overlap of the two surveys, the total amount of sky covered is approximately 29,700 square degrees.

In both surveys, most fields have been observed multiple times per season over many years. Figure 1 shows the field coverage with the gray scale indicating the number of seasons each field has been observed at least four times. The overall 

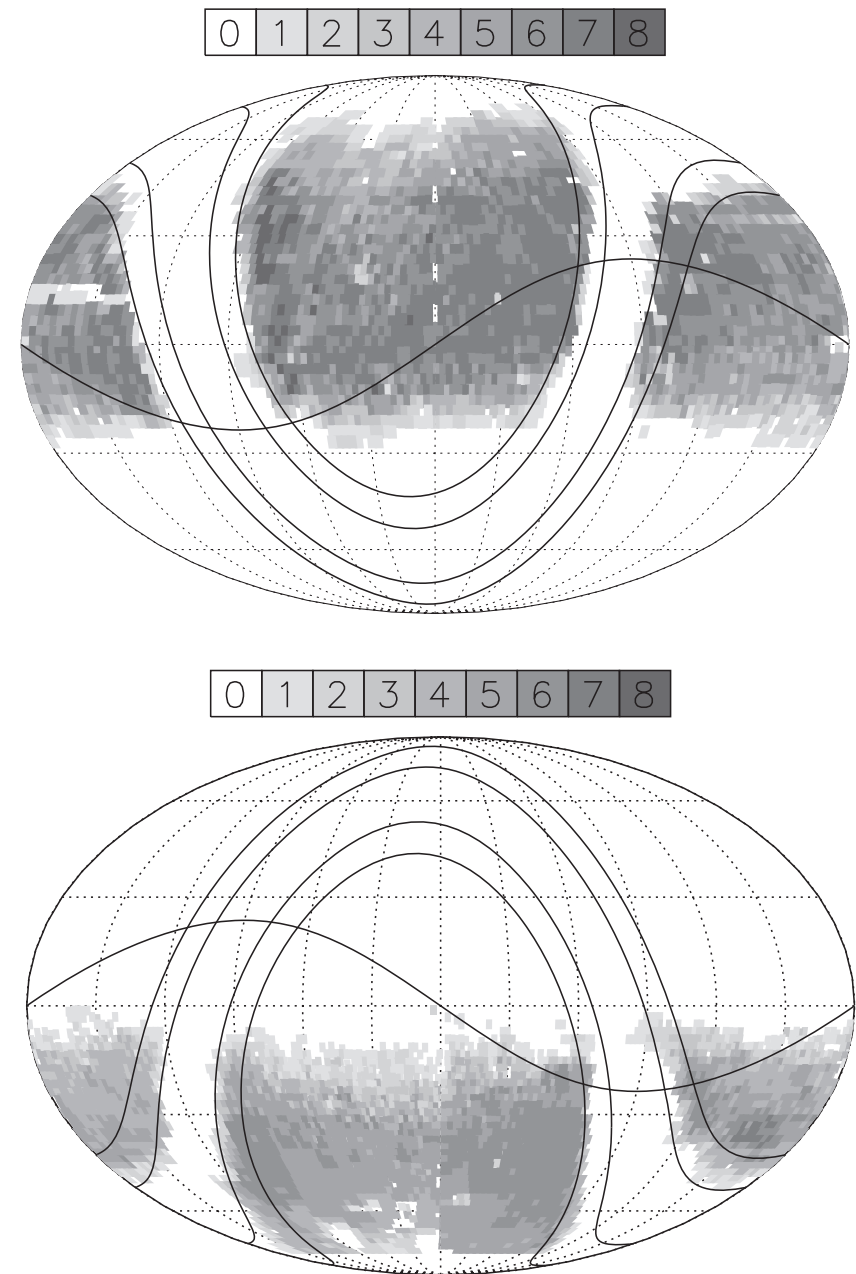

Figure 1. (a) Coverage of the Catalina Sky Survey (CSS). The shading shows the number of opposition seasons in which each field has been covered four or more times. The equal area plot is centered at an R.A. of $180^{\circ}$ and a decl. of $0^{\circ}$ with grid marks placed every $30^{\circ}$ of R.A. and decl. Contours of galactic latitude of $10^{\circ}$ and $20^{\circ}$ are shown as well as a line showing the ecliptic. (b) Coverage of the Siding Spring Survey (SSS). All parameters are as in (a) except that the plot is centered at an R.A. of $0^{\circ}$.

cadence of the surveys is extremely non-uniform; the four (or more) observations could be within a single lunation or could be spread over a six-month opposition season. As we will see below, however, these details have no effect on our detection scheme. The practical limit that we find is that an object needs to have been detected at least four times in a single season in order for us to extract it from the data set.

In addition to being searched for NEOs and other moving objects, the CSS and SSS data are analyzed by the Catalina Real-time Transient Survey (CRTS; Drake et al. 2009) to search for transients. The CRTS compares source catalogs from each image with source catalogs generated from deep coadds of multiple images in addition to comparing with other available deep catalogs; objects which do not appear in these catalogs are deemed candidate transients. CRTS performs additional filtering in an attempt to cull the true astrophysical transients from the large number of image artifacts. We begin our analysis, however, with the full catalog of candidate transients. The full CSS catalog has nearly 1.8 billion transient candidates from 2005 December 6 to 2012 April 21, while the SSS catalog has 2.1 billion candidates from 2005 February 20 to 2012 April 29.

\section{THE SLOWLY MOVING OBJECT SEARCH}

\subsection{Creation of the Transient List}

The cadence of the NEO surveys has allowed them to detect objects out to the orbit of Uranus. We will thus define our heliocentric radius of interest to be $25 \mathrm{AU}$ and beyond. An object in a circular prograde orbit at $25 \mathrm{AU}$ has a maximum retrograde motion at opposition of $4.9 \mathrm{arcsec} \mathrm{hr}^{-1}$. Motions higher than this would would likely be detected in the NEO surveys.

In typical operations, both the CSS and SSS take four images per field per night over a time interval of about 30 minutes. As our first step in our analysis, we require the detection of four transient candidates on a single night within a diameter defined by 4.9 arcsec times the maximum time interval. Note that we perform no other filtering here. The four detections are not required to show linear motion (as they would not for the slowest moving objects) or have similar measured magnitudes (which they might not at the magnitude limit of the survey). When four detections within an appropriate diameter are found, they are collected as a single transient with the average position, magnitude, and observation time of the four individual candidate transients.

Some fraction of the CSS and SSS transients recur at the same location. These transients are presumably some type of astrophysical source which has brightness variations sufficiently large that the source does not appear in the deep catalog but the object occasional becomes bright enough to appear in individual images. To remove these clear non-solar system sources, we search for all transients that have a transient detected on a different night within $4 \operatorname{arcsec}$ of the same location. Our final transient list, 1.2 million sources in the CSS fields and 2.3 million sources in the SSS fields, will contain all outer solar system objects within the geometric and brightness limits of the survey, true astrophysical transients which appear only once at their location, and image artifacts, which will be the overwhelming majority of the list.

Figure 2 shows the locations of each of these transients. Significant structure can be see in the transient locations. In the $\mathrm{SSS}$, in particular, transients occur frequently on the field edges, suggesting inconsistent astrometric solutions in these areas (which will lead stationary stars to occasionally be classified as transients in large numbers). Similar effects can be seen for the CSS data in the far north. Other regions of clear artifact can be seen. In addition, the higher density of transients in the SSS is clear. The SSS contains 163 transients per square degree compared to 61 in the CSS. This larger number of southern transients will make the SSS moving object search comparatively more difficult.

Nonetheless, real known Kuiper belt objects are also present in the data. As an example, Figure 3 shows the orbit of Makemake - the brightest known KBO after Pluto-as well as the transients that are detected in this region of the sky. Of these 789 transients, 53 are detections of Makemake itself. The other known bright KBOs likewise have many detections.

\subsection{The Keplerian Filter}

All objects in the outer solar system move on well-defined Keplerian orbits. We use this fact to look for collections of transients which define any physically possible Keplerian orbit. Three points in the sky at different times are required to define an orbit, but because the three points contain nine parameters 

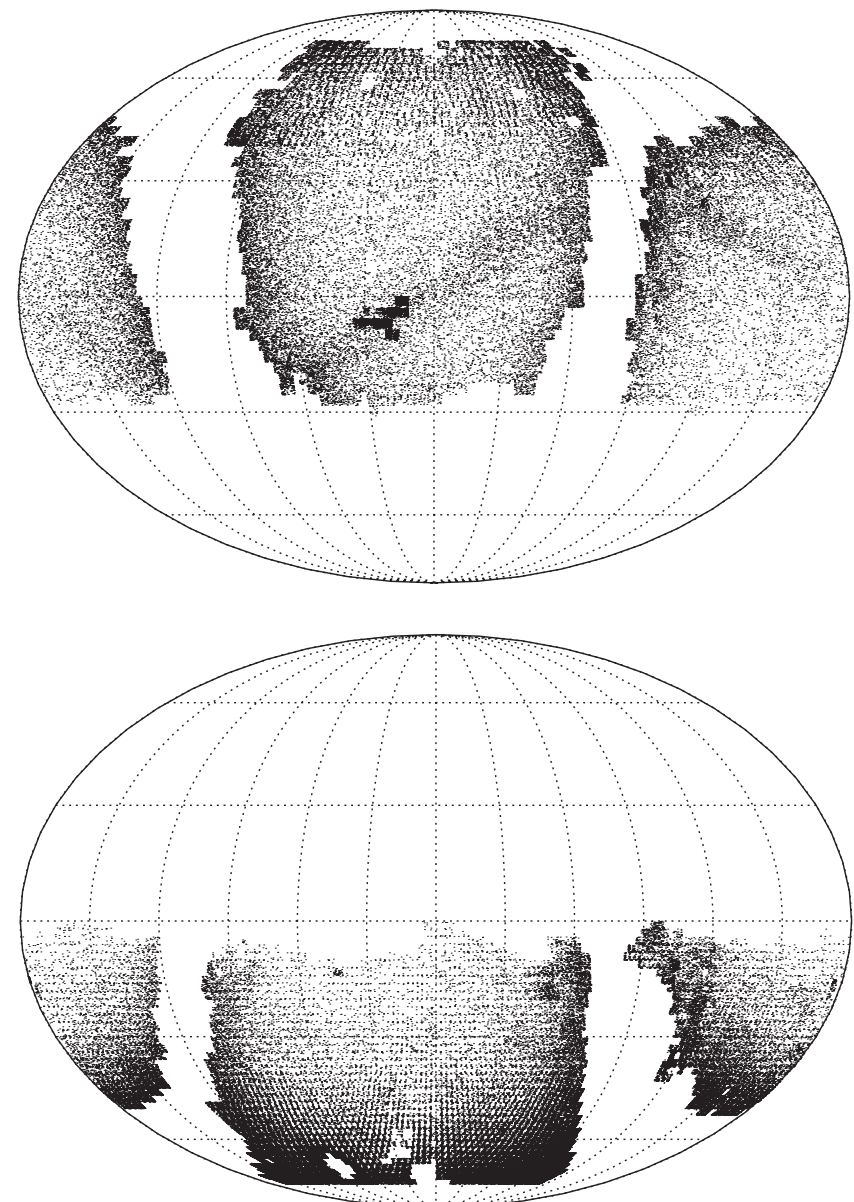

Figure 2. (a) Locations of transients in the CSS. To allow viewability only every 20th transient is shown. Geometry is as in Figure 1(a). (b) The locations of transients in the SSS. Every 20th transient is shown. Geometry is as in Figure 1(b).

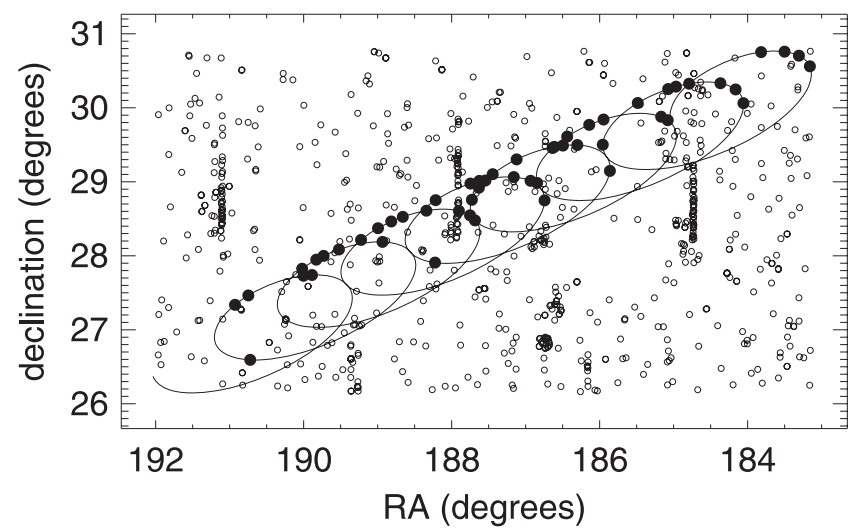

Figure 3. All transients detected in the vicinity of Makemake. The path of the orbit of Makemake over the 8 year period of the survey is shown as a solid line. The 53 times that Makemake was identified as a single-night transient are shown as filled circles. Significant structure is often seen in the transient locations, usually associated with the edge of a field or with artifacts from bright stars.

while an orbit is defined by only seven parameters, the three points overconstrain the orbit. Thus arbitrary sets of three points will not be able to be fit to a Keplerian orbit.

Fitting of orbits is a complex nonlinear problem. Attempting to fit all combinations of three points in the transient list $\left(\sim 10^{18}\right.$ attempted fits for CSS and $\sim 10^{19}$ for SSS) is computationally prohibitive, so we seek methods to minimize the number of orbital computations required. First, we will consider only combinations of transients with pairs of transients separated by no more than 120 days. This constraint is nearly, though not precisely, equivalent to requiring three transients observed over a single opposition season. As a short hand, however, we will refer to this constraint as requiring three transients over a single opposition.

If we confine ourselves to a single opposition season, we can also confine ourselves to a significantly smaller area of the sky. A stationary object at $25 \mathrm{AU}$ would have a $5^{\circ}$ parallax over a six month season. We conservatively allow motions up to $5^{\circ}$ for our 120 day maximum separation. This single season spatial and temporal filtering brings the number of potential orbital triplets that need to be checked down to 160 billion for CSS and 18 trillion for SSS. While this initial filter cuts the number of orbits to be fit significantly, even this number would be computational prohibitive for full Keplerian orbital fitting.

We apply one more filter which is appropriate for these distant objects observed over a modestly short time interval. As shown in Bernstein \& Khushalani (2000), motions of distant objects in the solar system can be approximated over a short time period as moving linearly through the solar system perpendicular to the earth-object vector. This sky plane approximation is defined by only six parameters: the motion vector in the plane of the sky, the distance to the object, and the position of the object at a single point in time. For each triplet of transients, we perform a least-squares fit for these six parameters. We then use the fitted parameters to calculate the predicted positions of the object at the times of observation and the residuals from this prediction.

To test the accuracy of this approximation, we examined the positions of real KBOs over single oppositions. As an example, if three observations are made of Makemake-at opposition, one month before, and one month after-the resulting linear approximation to the orbit predicts the position of Makemake to within 25 arcsec for the four months surrounding opposition. The approximation is the worst for the the nearest and most eccentric objects. But similar observations of the positions of 2005 EB299, for example-with an eccentricity of 0.51 and a semimajor axis of $52 \mathrm{AU}$, and currently near perihelion at 25.7 AU—predict the position to within 45 arcsec for the same period. For our Keplerian filter we conservatively require that the maximum residual between the linear sky-plane fit and the data is $50 \mathrm{arcsec}$ or less. In addition, we require that the heliocentric distance retrieved from the sky-plane approximation be larger than $10 \mathrm{AU}$ and that the orbital energy be within a factor of 1.5 of the maximum for a bound object at that distance. Experimentation with synthetic orbits suggests that these limits will detect real objects beyond $25 \mathrm{AU}$ in nearly all combinations of three observations in an opposition season. After applying this filter, we have 140 million triplets remaining in the CSS data and 3 billion in the SSS.

The final step in the Keplerian filter is full orbital fitting. We use the code of Bernstein \& Khushalani (2000) which efficiently calculates orbits including planetary perturbations for outer solar system objects. When the orbits of real KBOs are fit by this code, we obtain sub-arcsecond residuals. Again, to be conservative, we require that the orbit of a transient triplet, when fit by this code, yield residuals smaller than only 5 arcsec. In addition we require that the retrieved heliocentric 
Table 1

Measured Properties of the Detected Objects

\begin{tabular}{|c|c|c|c|c|c|c|}
\hline $\begin{array}{l}\text { Linked } \\
\text { Detections }\end{array}$ & $\begin{array}{l}V \text { mag } \\
\text { (avg) }\end{array}$ & $\begin{array}{c}a \\
(\mathrm{AU})\end{array}$ & $e$ & $\begin{array}{c}i \\
(\mathrm{deg})\end{array}$ & $\begin{array}{c}\text { Distance } \\
\text { (AU) }\end{array}$ & $\begin{array}{l}\text { Identified } \\
\text { Object }\end{array}$ \\
\hline 51 & $17.06 \pm .02$ & $45.501 \pm 0.003$ & $0.160509 \pm 0.00007$ & $29.002 \pm 0.001$ & $51.867 \pm .001$ & Makemake \\
\hline 47 & $17.37 \pm .01$ & $43.102 \pm .002$ & $0.195129 \pm 0.00009$ & $28.205 \pm 0.001$ & $51.217 \pm .001$ & Haumea \\
\hline 28 & $18.53 \pm 0.01$ & $67.82 \pm 0.02$ & $0.4384 \pm .0002$ & $43.992 \pm 0.001$ & $96.895 \pm .006$ & Eris \\
\hline 8 & $19.5 \pm 0.1$ & $43.21 \pm 0.03$ & $0.118 \pm 0.002$ & $25.855 \pm 0.001$ & $41.186 \pm 0.004$ & 2002 TX300 \\
\hline 4 & $19.2 \pm .1$ & $31 \pm 16$ & $0.4 \pm 0.5$ & $2.34 \pm 0.06$ & $30.37 \pm .06$ & Nereid \\
\hline
\end{tabular}

distance of the object be larger than $15 \mathrm{AU}$. We put no other orbital constraints on the fit.

Full Keplerian filtering yields 4.8 million triplets in the CSS data and 235 million triplets in the SSS data which can be fit to Keplerian orbits.

\subsection{Further Filtering}

Keplerian filtering yields impressive results. For the CSS, for example, out of $10^{19}$ possible combinations of three transients observed across the whole sky over a seven-year period, only one out of every 2 trillion could possibly be fit to single-season Keplerian orbits for objects in the solar system beyond $25 \mathrm{AU}$. Nonetheless, these triplets are predominantly not real objects, but rather chance alignments of astrophysical transients or noise, so further filtering is needed.

There are many potential methods we could use to further filter the transients. We could, for example, require that all transients in a triplet have the same magnitude within limits, or we could tighten the constraints on the final orbit fitting. But because of our large data set with the potential that any real object will be observed many times, we chose instead to require that there be additional observed transients which also fit the same orbit.

The simplest requirement to implement is that an orbit be required to have four, rather than three, observations during an opposition season. The large number of fields in which observations have been made four or more times in a season (Figure 1) suggests that this method could be quite efficient at detecting objects.

To find quadruplets, rather than just triplets, which fit a Keplerian orbit, we simply note that any real quadruplets will be made up of combinations of the triplets that we have already collected. We examine all of the triplets that already passed our initial Keplerian filter and we look for pairs of triplets in in which two of the three transients in the triplet are common across the pair, thus defining four unique transients. We pass these quadruplets of transients through the Bernstein \& Khushalani (2000) orbit fitting routine and retain any quadruplets for which the calculated $\chi^{2}$ of the fit is below 10. We find 1192 good quadruplet fits to Keplerian orbits in the CSS data and 5515 in the SSS data. These numbers are sufficiently small that we now examine the results in detail.

\section{RESULTS}

\subsection{CSS}

Examining the locations of CSS quadruplets we see that the majority are tightly clumped into a few distinct locations in the

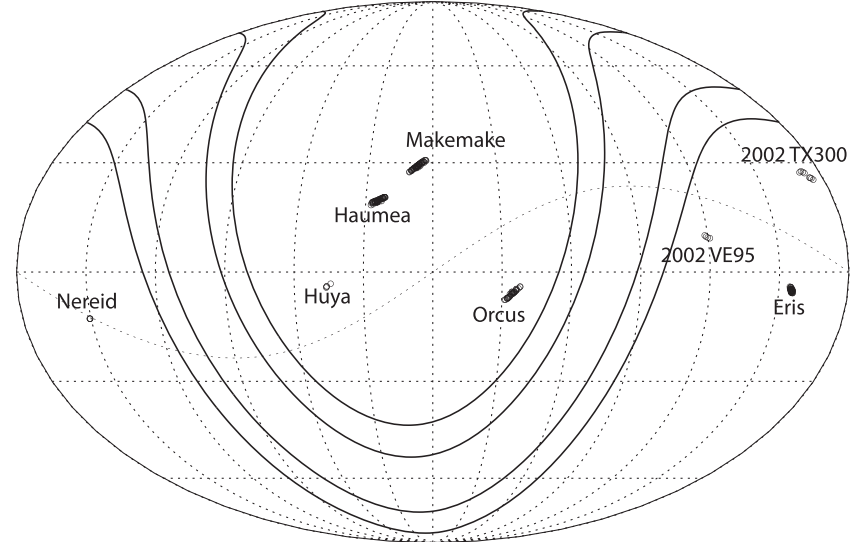

Figure 4. Eight objects detected in our survey. Each of the objects is found to be a previously discovered object in the outer solar system. The geometry is as in Figure 1(a).

sky (Figure 4). For each quadruplet we examine if other quadruplets fit the same orbit by again running the full orbit fit through the (Bernstein \& Khushalani 2000) routine. In this manner we find that the 1192 CSS quadruplets define 8 distinct objects (Table 1). Each of these recovered objects is, in fact, a known bright object in the outer solar system. Requiring only four detections within an opposition season reduces the false positive rate in this data set to zero.

While we have no rigorous method of assessing the detection efficiency of this survey, we estimate the efficiency by examining the detections of the brightest known objects. Table 2 shows the brightest known objects in the solar system beyond $25 \mathrm{AU}$ (with the exception of Uranus and Neptune, which saturate in our survey). Looking at the initial unfiltered list of transients, we determine how many times each object was detected as a transient in a survey, and, furthermore, how many separate oppositons were linked. Our algorithm was $100 \%$ efficient at recovering known objects when four or more detections were available in an opposition.

All known outer solar system objects brighter than $V=19.1$ were detected with only two exceptions. Pluto is in a region of the galactic plane which was never observed. Quaoar was in the survey region for only the first season when only two images were obtained. In later seasons Quaoar moved into the galactic avoidance zone. Nereid, an irregular satellite of Neptune which does not follow a heliocentric Keplerian orbit, was detected 14 times and recovered during an opposition season when its orbit was indistinguishable from a heliocentric orbit. The bright outer solar system objects with $V \lesssim 19.1$ were all not just detected, they were observed and would have been detected 
Table 2

The Brightest known Objects in the Outer Solar System

\begin{tabular}{|c|c|c|c|c|c|c|}
\hline Name & $\begin{array}{c}V \text { mag } \\
(\text { avg })\end{array}$ & $\begin{array}{c}\text { Distance } \\
(\mathrm{AU})\end{array}$ & $\begin{array}{c}a \\
(\mathrm{AU})\end{array}$ & $e$ & $\begin{array}{c}\text { Inc } \\
(\mathrm{deg})\end{array}$ & Notes \\
\hline 134340 Pluto & 14.0 & 31.6 & 39.4 & 0.25 & 17.2 & not in survey region \\
\hline 136472 Makemake (2005 FY9) & 16.9 & 52.1 & 45.45 & 0.16 & 29.0 & 53 detections, 8 oppositions linked \\
\hline 136108 Haumea (2003 EL61) & 17.3 & 51.1 & 43.17 & 0.19 & 28.2 & 49 detections, 6 oppositions linked \\
\hline 136199 Eris (2003 UB313) & 18.7 & 96.7 & 67.71 & 0.44 & 44.2 & 39 detections, 6 oppositions linked \\
\hline Nereid & 18.8 & 30.0 & 30.1 & 0.01 & 1.76 & 14 detections, 1 opposition linked \\
\hline 50000 Quaoar (2002 LM60) & 18.9 & 43.2 & 43.58 & 0.04 & 8.0 & not in survey region \\
\hline $55636(2002$ TX300) & 19.6 & 41.5 & 43.10 & 0.12 & 25.9 & 17 detections, 2 oppositions linked \\
\hline 28978 Ixion (2001 KX76) & 19.6 & 41.7 & 39.59 & 0.24 & 19.6 & no detections; low galactic latitude \\
\hline 230965 (2004 XA192) & 19.7 & 35.9 & 46.90 & 0.24 & 38.1 & not in survey area \\
\hline 38628 Huya (2000 EB173) & 19.7 & 28.8 & 39.77 & 0.28 & 15.5 & 19 detections, 1 opposition linked \\
\hline 120178 (2003 OP32) & 19.9 & 41.4 & 43.03 & 0.11 & 27.2 & 1 detection \\
\hline (2010 EK139) & 20.0 & 39.9 & 70.26 & 0.54 & 29.4 & 1 detection in SSS \\
\hline 20000 Varuna (2000 WR106) & 20.1 & 43.4 & 42.91 & 0.05 & 17.2 & 2 detections \\
\hline 47171 (1999 TC36) & 20.1 & 30.8 & 39.31 & 0.22 & 8.4 & 5 detections, max of 2 in single opposition \\
\hline 145452 (2005 RN43) & 20.1 & 40.7 & 41.37 & 0.02 & 19.3 & 4 detections, max of 2 in single opposition \\
\hline 229762 (2007 UK126) & 20.1 & 45.5 & 73.06 & 0.49 & 23.4 & 4 detections, max of 1 in single opposition \\
\hline 55637 (2002 UX25) & 20.2 & 41.8 & 42.55 & 0.14 & 19.5 & 7 detections, max of 3 in single opposition \\
\hline 278361 (2007 JJ43) & 20.2 & 41.8 & 48.21 & 0.16 & 12.1 & too far south for CSS; no detections in SSS \\
\hline 55565 (2002 AW197) & 20.3 & 46.6 & 47.54 & 0.13 & 24.3 & 2 detections \\
\hline 174567 Varda (2003 MW12) & 20.3 & 47.9 & 45.85 & 0.14 & 21.5 & 1 detection \\
\hline 55638 (2002 VE95) & 20.4 & 28.4 & 39.22 & 0.29 & 16.4 & 12 detections, 1 opposition linked \\
\hline 303775 (2005 QU182) & 20.4 & 47.9 & 110.28 & 0.67 & 14.0 & 0 detections \\
\hline$(2004$ NT33) & 20.4 & 38.1 & 43.41 & 0.15 & 31.2 & not in survey region \\
\hline 202421 (2005 UQ513) & 20.4 & 48.8 & 43.22 & 0.15 & 25.7 & no detections \\
\hline
\end{tabular}

independently multiple times. The least well-observed object of this brightness was Orcus. It was independently detected 381 times as a Keplerian quadruplet. It was detected at each opposition in which four or more observations were made of its region. Any one of these detections would have sufficed. The other bright objects were independently detected comparable or greater numbers of times. It appears that for bright objects, where detection is not limited by signal-to-noise ratio, our algorithm is essentially $100 \%$ efficient at detecting objects when a sufficient number of observations is obtained. Because of the nature of the data, which are simply reported detections of transients, we cannot rigorously prove this assertion of $100 \%$ algorithmic efficiency through population modeling, for example, as is often done in surveys. We instead point out that the real known objects in the sky provide, essentially, a model population with thousands of independent quadruplet test cases. Our algorithm correctly recovers each of these independent combinations of real object observations with $100 \%$ efficiency when there are four or more detections in a single opposition season. We conclude that for sufficiently bright objects in regions with good coverage, we will lose zero objects.

The next faintest objects detected, 2002 TX300 and Huya, with predicted $V \sim 19.6$ and measured $V \sim 19.4$, each have more than a dozen individual detections, but are only detected four times in an opposition season twice and once, respectively.
It is clear that objects of this magnitude will occasionally be missed not because of algorithmic inefficiencies but simply because they will not always be detected even when they are in the observed field.

Between $19.5<V<20.1$ many objects were detected three or fewer times per opposition season but only 2002 VE95 (with a measured magnitude of $V=19.8$ ) has a single four-detection opposition. Even with just this single opposition season in which 2002 VE95 is detected, the algorithm correctly identifies 2002 VE95 in the data.

We conclude that the detection efficiency must be nearly $100 \%$ for $V \lesssim 19.1$ for regions well covered in the survey and it must begin dropping around $V \sim 19.4$ until it reaches zero by $V \sim 20$. While the precise shape of the efficiency cannot be defined, the general shape and behavior appears clear.

\section{2. $S S S$}

A total of 5515 Keplerian-fitting quadruplets are found in the SSS survey. None of these quadruplets can be linked to another quadruplet, thus it appears that these are likely all false positives. With the significantly higher number density of detected transients in the SSS fields, requiring four detections is insufficient for removing all of the false positives. Clearly, since none of the quadruplets can be linked, adding that requirement that an object be linked four times in each of two opposition seasons reduces the false positive rate to zero. But 
we also find that a less stringent requirement-that we link the object five times in one opposition rather than just four timesis also sufficient to drop the false positive rate to zero. It is possible, of course, that 1 or more of the 5515 quadruplets is a real object that is sufficiently faint to only have 4 detections and which is removed by the more stringent filtering. This possibility demonstrates that the addition of a more stringent detection criterion lowers our true detection threshold in this part of the survey. With no effective way to perform followup observations of candidates, such a lowering of the detection threshold is necessary in order to remove false positives.

Determining an efficiency for the SSS is more difficult with a lack of detections of real objects, but we make an estimate based on the experience with the CSS. First, the SSS images are about 0.5 mag less deep than CSS images. Second, the requirement of five detections raises the detection threshold. While a five detection requirement would have detected the $V \sim 19.4$ objects in the CSS, all fainter objects would have been missed. We thus estimate that our detection efficiency is nearly $100 \%$ for $V \lesssim 18.6$ and begins to drop by $V \sim 18.9$. We have no reliable method of determining where the efficiency drop to zero but we suspect it happens quickly faintward of $V=18.9$.

\section{DISCUSSION}

No new bright outer solar system objects were detected in this all sky survey to an approximate limit of $V=19.4$ in the northern survey and an estimated limit of $V=18.9$ in the southern survey. If any bright objects remain to be discovered in the outer solar system they must be at extreme ecliptic latitudes or close to the galactic plane. No bright objects in the outer solar system have been discovered with inclinations higher than the $44^{\circ}$ inclination of Eris, so we do not anticipate any undiscovered objects at the ecliptic poles.

We estimate the probability that any bright objects remain to be discovered. The sky density of bright objects appears approximately uniform within $30^{\circ}$ of the ecliptic, while no bright objects have been found at higher latitudes (Schwamb et al. 2014). Our survey covered $80 \%$ of the sky within $30^{\circ}$ of the ecliptic. The uncovered $20 \%$ is located within $20^{\circ}$ of the galactic plane. The survey of Sheppard et al. (2011) covered approximately one third of the galactic plane below ecliptic latitudes of $30^{\circ}$ to a depth of approximately $R=21$ with a completeness of approximately $75 \%$. There are six known objects brighter than $V \sim 19$, four of which were in our survey region and one recovered in the galactic plane survey.
From these surveys, we estimate that the probability that there is one or more remaining objects in the outer solar system brighter than $V \sim 19$. To do so we construct Monte Carlo models of $\mathrm{KBO}$ populations with a varying sky densities. Selecting the simulation which are compatible with the detections of the two data sets, we find that the probability that there is one additional object yet to be detected is $32 \%$. The probability that there are two or more is $10 \%$. For all regions except for the galactic plane, these limits extend to very distant objects; a body at 10,000 AU, for example, would still move $\sim 10$ arcsec in a month at opposition and would be detected in our analysis.

Schwamb et al. (2014) estimate that surveys of the outer solar system have been approximately $70 \%$ complete to $R=19.5$. This survey suggests that at the brightest end the surveys to date have been even more efficient and that the most likely scenario is that no new bright objects remain to be discovered.

In addition to demonstrating the only modest probability of the existence of additional bright objects in the outer solar system, this survey demonstrates the relative ease of detecting slowly moving solar system objects in transient surveys.

This search for moving objects in the CRTS catalog has been supported by grant NNX09AB49 G from the NASA Planetary Astronomy program. The CRTS survey was supported by the NSF grants AST-0909182, AST-1313422, and AST-1413600. The CSS survey is funded by the National Aeronautics and Space Administration under Grant No. NNG05GF22 G issued through the Science Mission Directorate Near-earth Objects Observations Program. This serendipitous survey was conceived during a serendipitous conversation at the LSST "All Hands" meeting between MEB and MJG.

\section{REFERENCES}

Bernstein, G., \& Khushalani, B. 2000, AJ, 120, 3323

Brown, M. E. 2008, in The Solar System Beyond Neptune, ed. M. A. Barucci et al. (Tucson, AZ: Univ. Arizona Press), 335

Drake, A. J., Djorgovski, S. G., Mahabal, A., et al. 2009, ApJ, 696, 870

Larson, S., Beshore, E., Hill, R., et al. 2003, BAAS, 35, 982

Rabinowitz, D., Schwamb, M. E., Hadjiyska, E., \& Tourtellotte, S. 2012, AJ, 144,140

Schwamb, M. E., Brown, M. E., \& Fraser, W. C. 2014, AJ, 147, 2

Schwamb, M. E., Brown, M. E., \& Rabinowitz, D. L. 2009, ApJL, 694, L45

Schwamb, M. E., Brown, M. E., Rabinowitz, D. L., \& Ragozzine, D. 2010, ApJ, 720, 1691

Sheppard, S. S., Udalski, A., Trujillo, C., et al. 2011, AJ, 142, 98

Trujillo, C. A., \& Brown, M. E. 2003, EM\&P, 92, 99 